\title{
The association between hip muscle cross-sectional area, muscle strength and bone mineral density.
}

${ }^{1}$ Harbeer Ahedi, ${ }^{1}$ Dawn Aitken, ${ }^{2}$ David Scott, ${ }^{1}$ Leigh Blizzard, ${ }^{3}$ Flavia Cicuttini,

${ }^{1}$ Graeme Jones.

${ }^{1}$ Menzies Research Institute of Tasmania, University Of Tasmania, Hobart 7000, Tasmania, Australia

${ }^{2}$ NorthWest Academic Centre, The University of Melbourne, St Albans, Victoria, Australia

${ }^{3}$ Department of Epidemiology and Preventive Medicine, School of Public Health and

Preventive Medicine, Monash University, Alfred Hospital, Melbourne, VIC 3004, Australia

${ }^{1}$ Harbeer Ahedi is supported by an Australian Government Australian Postgraduate Award.

${ }^{1}$ Dawn Aitken is supported by an NHMRC Early Career Fellowship.

${ }^{1}$ Leigh Blizzard is supported by an NHMRC Career Development Fellowship.

${ }^{1}$ Graeme Jones is supported by an NHMRC Practitioner Fellowship.

\section{Address correspondence to:}

Harbeer Ahedi,

Menzies Research Institute Tasmania, University of Tasmania,

Private Bag 23, Hobart, Tasmania 7000, Australia

Phone +61 362264232

Fax: +61 362297704

Email: $\underline{\text { Harbeer.Ahedi@utas.edu.au }}$ 


\title{
Mini abstract/summary
}

This population-based study investigates the association between BMD, muscle size and strength. Hip flexors and, to a lesser extent, hip rotators were correlated with BMD of the hip and femoral neck and this relationship was stronger for women. Hip muscle size was associated with muscle strength while muscle strength showed a weak association with BMD.

\begin{abstract}
Purpose: Studies examining the association between muscle size, muscle strength and bone mineral density (BMD) are limited. Thus, this study aims to describe the association between hip muscles cross-sectional area (CSA), muscle strength and BMD of the hip and spine.
\end{abstract}

Methods: 321 subjects from the Tasmanian Older Adult Cohort (TASOAC) study with a right hip MRI scan conducted between 2004-2006, were included. Hip muscles were measured on MR images using OsiriX (Geneva) software measuring maximum muscle CSA $\left(\mathrm{cm}^{2}\right)$ of gluteus maximus, obturator externus, gemelli, quadratus femoris, piriformis, pectineus, sartorius and iliopsoas. Dual-energy x-ray absorptiometry (DXA) measured total hip, femoral neck and spine BMD and lower limb muscle strength was assessed by dynamometer.

Results: Muscle CSA of the hip flexors (pectineus, sartorius and iliopsoas) and the hip rotators; obturator externus and quadratus femoris were associated with both total hip and femoral neck BMD (all $p<0.05$ ). The associations between CSA of pectineus and sartorius and BMD were stronger in women $(p=0.01-0.001)$ compared to men $(p=0.12-0.54)$. Additionally, only gemelli CSA was associated with BMD of the spine $(p=0.002)$. Gluteus maximus and piriformis showed no relationship with BMD. CSA of most hip muscles (except gluteus maximus and gemelli) were positively associated with leg strength $(p=0.02-<0.001)$. Lastly, leg strength was weakly associated with $\operatorname{BMD}(p=0.11-0.007)$.

Conclusion: Hip muscle CSA, and to a lesser extent, muscle strength were positively associated with hip BMD. This data suggests that both higher muscle mass and 
strength may contribute to the maintenance of bone mass and prevention of disease progression in older adults.

\section{Introduction:}

Loss of skeletal muscle mass and strength, also known as sarcopenia, has been recognized as a predictor of reduced muscle strength and bone mineral density (BMD) $[1,2]$, possibly due common determinants $[3,4]$. Additionally, studies have found that subjects with lower skeletal mass are at higher risk of falls [5], fractures [6] and loss of function [7]. Muscle cross-sectional area (CSA) is a validated surrogate measure for muscle mass. Research suggests that CSA of the thigh in the elderly predicts hip fractures [8] and increased risk of osteoporosis [9]. Similarly, individual muscle CSA associates with cartilage $[10,11]$, muscle strength $[12,13]$, joint biomechanics [14] and bone structure $[15,16]$.

In comparison to skeletal mass, fewer studies have investigated the correlations between individual muscle CSA and BMD. For instance, Revel et.al compared the associations between psoas, erector spine and triceps brachii muscle CSA and BMD of lumbar spine in 89 post-menopausal women and found a correlation between psoas muscle CSA and spine BMD [17]. The same group conducted a clinical trial in which 67 post-menopausal women were selected for physical training targeting either the psoas or the deltoid muscles. After 12 months, women who had trained their deltoid muscle had a greater loss in spine BMD in comparison to women who had trained their psoas muscle [-8.87\% $\mathrm{v}+0.14 \%]$ [18]. These studies suggest that local muscles, which insert directly into the bone, are involved in the preservation of bone density. Furthermore, in a magnetic resonance imaging (MRI)-based cross-sectional study, Klein et.al suggested that both upper arm muscle CSA and forearm muscle CSA were the best predictors of humerus and forearm cortical bone health [16]. Lastly, in a longitudinal study using peripheral quantitative computed tomography (pQCT), Edwards et.al found larger forearm CSA was positively associated with higher radial bone mineral content and bone area but not bone density[15]. These studies support the Wolf and Frost theory [19, 20], which proposes, that the increase in bone mass is due to muscle accumulation on bone and muscle recruitment and contraction during locomotion. 
Skeletal muscle is not only linked with bone mass but also influences variability in strength $[14,21]$. Although muscles have been examined as groups; studies investigating the relationship between individual muscles and strength are rare. Masuda et.al investigated the correlation of isokinetic muscle strength and muscle CSA of the lower limb in 14 healthy soccer players (age 19-22 yrs.) and reported that CSA of gluteus medius and minimus (scored together) and gluteus maximus were strongly correlated with hip abductor strength. Additionally iliopsoas muscle CSA was moderately correlated with hip flexion strength [13]. Takai et.al, conducted a cross-sectional study in older adults (age 63-64 yrs.) and found a very strong correlation between quadriceps muscle CSA and isometric knee extension force during sit-to-stand testing [22]. Furthermore, Frontera et.al investigated the longitudinal changes in muscle CSA and muscle strength in 9 men (age 65yrs.) for 12 years and concluded that variation in muscle CSA was accountable for $90 \%$ of variability in muscle strength.

In general, association between muscle mass and measured individually or as a group is positively associated with BMD and strength. However, firstly, less is known about the relationship of individual muscles and bone mass. Furthermore, studies suggest that muscles having a direct link with the joint and those which are recruited often, might have a greater influence on bone mass. Secondly, examining the relationship between muscle CSA and muscle strength provides greater understanding of muscle morphology and also aids in maintaining muscle strength in older adults. Thus, the objective of our study was to describe the cross-sectional associations between individual hip muscles, muscle strength and bone density at the hip, femoral neck and spine.

\section{Methods and Materials:}

\section{TASOAC cohort}

This study was conducted as a part of the Tasmanian Older Adult Cohort (TASOAC) study, a prospective, population-based study initiated in 2002 aimed at identifying the environmental, genetic and biochemical factors associated with the development and progression of OA at multiple sites (hand, knee, hip and spine). Subjects between the 
ages of 50 to 80 years were randomly selected from the electoral roll of Southern Tasmania (population 229,000), with an equal number of men and women. The overall response rate was $57 \%$. As TASOAC was designed to examine communitydwelling older adults and hence all institutionalized older adults were excluded. Participants were also excluded if they had a contraindication for MRI, including previous hip replacements. Of all initially eligible participants, 1,100 enrolled in the study, and 1,099 attended a baseline (Phase 1) clinic between March 2002 and September 2004. Follow-up data (Phase 2) was collected for 875 participants at a clinic approximately three years later, and then again for 769 participants (Phase 3) at a clinic approximately five years later.

Hip MRI scans were added to the study in the later stages of phase 2. 328 subjects had a right hip MRI (T1-weighted) at phase 2 and of these 7 subjects had missing data or corrupted MRIs leaving 321 with complete data. No subject included in this study had a history of hip fracture at phase 2. In addition, at baseline (Phase 1) subjects included in the current study $(\mathrm{n}=321)$ were younger (age: $61.6 \mathrm{v} 63.6, \mathrm{p}<0.001)$ in comparison to the rest of the TASOAC cohort $(\mathrm{n}=778)$, however no other differences in sex, BMI, bone mineral density and leg strength were found between the two populations. Written informed consent was obtained from all participants and the Southern Tasmanian Health and Medical Human Research Ethics Committee approved this study.

\section{Clinical measurements:}

Demographic characteristics, medical history and lifestyle factors were assessed by self-administered questionnaires. Height was measured to the nearest $0.1 \mathrm{~cm}$ using a stadiometer (with shoes, socks and bulky clothes removed). Weight was measured to the nearest $0.1 \mathrm{~kg}$ (with shoes, socks and bulky clothes removed) using a single pair of electronic scales (Seca Delta model 707; Hamburg, Germany). Body mass index (BMI) was calculated as the weight $(\mathrm{kg})$ divided by height $\left(\mathrm{m}^{2}\right)$.

\section{Magnetic Resonance imaging:}

An MRI scan of the right hip was performed. The hip was imaged in the sagittal plane using a 1.5 Tesla G.E signal whole-body magnetic resonance unit with a phased-array flex coil. The following image sequence was used: a T1-weighted fat-suppressed 3- 
dimensional gradient-recalled acquisition in the steady state; flip angle $55^{\circ}$; repetition time $58 \mathrm{msec}$; echo time $12 \mathrm{msec}$; field of view $16 \mathrm{~cm}$; 60 partitions; 512 × 512-pixel matrix; acquisition time 11 minutes 56 seconds, and 1 acquisition. Sagittal images were obtained at a partition thickness of $1.5 \mathrm{~mm}$ and an in-plane resolution of $0.39 \mathrm{x}$ $0.39 \mathrm{~mm}(512 \times 512$ pixels $)$. In this study the approximate range of hip MRI images was from the middle of greater sciatic notch in the pelvis to the end of the lesser trochanter of the femur.

\section{Measurement of muscle cross-sectional area (CSA):}

Hip muscles were identified and chosen for measurement as per MRI field of view. Measurements of clearly visible muscle CSA, where the entire area of the muscle was visible and distinguishable from the adjacent muscles were made at the anatomical landmarks described in Table 1. Hip muscle area was assessed on MR images using OsiriX (Geneva) software measuring maximum muscle CSA $\left(\mathrm{cm}^{2}\right)$ of gluteus maximus, obturator externus, gemelli, quadratus femoris, piriformis, pectineus, sartorius and iliopsoas (Figure 1). If any of the hip muscles above were not distinguishable from adjacent muscles they were not measured; hence not all eight muscles were measured in all subjects. The CSA of each hip muscle was measured on two consecutive slices by one trained observer (HA) and the average was taken as the final measurement. The majority of hip muscles were measured on sagittal MR images except iliopsoas, which was measured by reformatting the whole sagittal image to the axial plane. The superior and inferior gemelli were measured together. The intra-class correlation coefficient (ICC) for the retest-reliability after two weeks ( $n=40$, same observer) was calculated for each muscle using the ICC $(3,1)$ formula. The ICCs for all hip muscle CSAs ranged from 0.98-0.99.

\section{Bone mineral density:}

Bone density $\left(\mathrm{g} / \mathrm{cm}^{2}\right)$ was assessed using dual-energy X-ray absorptiometry (DXA) at the right total hip, femoral neck and spine using a single Hologic Delphi densitometer (Waltham, MA, USA). The longitudinal coefficient of variation for our densitometer using a spine phantom was $0.39 \%$ [23] 


\section{Muscle strength:}

Leg strength (Figure 2) was measured to the nearest kilograms in both legs simultaneously, using a dynamometer (TTM Muscular Metre, Tokyo, Japan). This test examines isometric strength, predominantly of the quadriceps and hip extensors, and has been described in detail previously [24].

\section{Physical activity:}

Physical activity was assessed as steps/day determined by (Yamax SW-200, Yamax USA, San Antonio, Texas, USA). Each participant was instructed to wear the pedometer for seven consecutive days. This was repeated 6 months later for seasonal variations. Mean steps/day was calculated as the average of the days worn at both time points.

\section{Statistical analysis:}

The characteristics of the subjects are summarized as means and standard deviations (SD), and for men and women separately because of the sex-related differences in muscle CSA. Associations between bone mineral density at the hip; femoral neck, and spine and muscle CSA are summarized as standardized regression coefficients. In order to test whether the association between muscle and spine BMD was independent to local BMD, the analysis for spine BMD was adjusted for hip BMD. The associations of hip and femoral neck BMD with pectineus and sartorius CSA varied by sex as determined by tests of statistical interaction, and are presented for men and women separately. Regression analyses were also conducted with muscle strength as the response variable, and in this case the results of association with iliopsoas are presented for men and women separately, based on significant interaction. Lastly, regression models were used to examine the association between muscle strength and bone density. All statistical models were adjusted for age, sex (where appropriate) and steps per day but not body size as muscle size was a key focus of this study. All statistical tests were two sided and $\mathrm{p}$ values $<0.05$ were considered significant. All statistical analysis was conducted using Intercooled Stata 12 for Mac (Stata Corp. College station, TX, USA) 


\section{Results:}

Table 2 describes the characteristics of the study sample. There was similar mean age and mean BMI in men and women, however, men had higher mean muscle strength, muscle CSA, steps per day and bone mineral density of hip, femoral neck and spine.

Table 3 shows the associations between bone density and muscle CSA as standardized regression coefficients. Apart from pectineus and sartorius, data for males and females are combined, as there was no interaction. For pectineus and sartorius CSA, the associations with bone density were stronger for women than for men.

Both obturator externus and quadratus femoris CSA were positively associated with hip and, to a lesser extent, femoral neck BMD, but CSA of piriformis and gluteus maximus showed no association with BMD at any site. The associations of all hip flexors with bone density of hip and femoral neck were similar and statistically significant. Lastly, of all the hip muscles, only gemelli CSA was associated with spine BMD.

Table 4 presents the associations between hip muscles CSA and muscle strength. All hip muscles except gemelli and gluteus maximus were positively but weakly associated with muscle strength.

Lastly, hip BMD was associated with muscle strength (Beta: 0.12 95\%CI 0.04, 0.20). Additionally, spine BMD showed a weak association with muscle strength (Beta: 0.10 95\%CI: 0.00, 0.21) and the association between femoral neck BMD and muscle strength (Beta: 0.07 95\%CI: -0.00, 0.15) did not reach statistical significance.

\section{Discussion:}

This is the first population-based study describing the associations between hip muscles CSA, muscle strength and bone density. In the present study, all hip flexor CSAs were associated with bone density and of these, associations for pectineus and sartorius were stronger for women in comparison to men. Among the hip rotators, 
obturator externus and quadratus femoris was associated with hip and femoral neck BMD. Gemelli was the only hip muscle that associated with spine BMD. Lastly, most of the hip muscles were associated with muscle strength and overall, muscle strength was weakly correlated with BMD.

In general all hip muscles were positively associated with bone density of the hip, femoral neck and spine. We adjusted for age and sex but not for body size. We acknowledge that this could lead to bias but we believe that as both, muscle size and BMD correlate with body size, any adjustment for body size would be an over adjustment. For instance, when we adjusted for BMI in the analyses between total hip BMD and muscle CSA, we noted a $2 \%$ to $63 \%$ reduction in the standardized regression coefficients (average of 44\% change). This change occurs because of the strong correlation between muscle CSA and BMI. Thus, the issue with adjustment for BMI or any mass related factor is that it may also reduce any genuine association between muscle size and BMD.

Most of the hip muscles, especially hip flexors, were associated with hip and femoral neck BMD but these associations differed in men and women. Of the hip rotators, quadratus femoris and obturator externus showed a weak association with BMD of hip and femoral neck but no difference by gender was found. The reasons for this are unclear. The literature on the relationship between skeletal muscle mass and bone density in men and women is controversial $[3,6,15,25-27]$. The available data does not allow clear conclusions due to differences in methodology, subjects and study designs. One possible explanation is that there are higher flexion and greater hip contact forces in women in comparison to men [28-30]. Thus it could be speculated that due to higher flexion and greater hip contact forces the relationship between bone density and hip flexion is stronger in females, however we did not measure biomechanical factors in this study.

Unexpectedly, some muscles did not show any associations with bone density or strength. For instance, no association was found between gluteus maximus size, muscle strength and bone density. This muscle is known to associate with muscle strength [13] but its association with bone density is currently unknown. These irregularities could be explained by our inability to assess the maximal muscle bulk 
due to limited MRI field of view (especially for the gluteus maximus muscle although it should be noted that the measured CSA was greater than any of the other measured muscle). An alternative potential explanation as to why gluteus maximus CSA was not associated with muscle strength is that other hip extensor muscles, which were not assessed in this study, may have been recruited preferentially to this muscle during the strength assessment.

Studies show that piriformis is activated during exercise and is mainly involved in hip rotation and does contribute to hip strength and stability [31]. In the present study, piriformis was weakly associated with muscle strength but not with bone density. Our findings support this fact, however like, gluteus maximus, the relationship of piriformis with bone density is unknown and nor does it associate with hip cartilage volume in subjects with or without hip OA [11]. Thus, evidence suggests that preservation of piriformis may result in better hip stability and perhaps this muscle has no role in maintenance of bone mass.

Skeletal muscle mass has been found to be associated with hip and spine BMD [3], however in this study the correlations between hip muscle CSA and BMD were mostly local (i.e hip and femoral neck) with the exception of gemelli, which was associated with spine BMD. Gemelli, is a deep hip rotator and has been proposed to play a vital role in hip/pelvic stability [32]. For instance, when hip rotators were spared during surgery, hip dislocation and function deficit rates dropped dramatically [33] and one study reports that gemelli CSA was positively associated with hip cartilage volume in subjects without hip OA $(\mathrm{p}=0.02)$ [11]. Thus, gemelli may play a role in osteoarthritis disease progression however its association with spine BMD is unclear. Nevertheless, it has been suggested that gemelli may fuse with its neighboring muscle, obturator internus and mimic its functions [34]. Obturator internus play's a vital role in stabilization of the pelvis, which is interdependent on the spine for its stability. Thus, we could speculate that due to its association with obturator internus, gemelli might show an association with spine BMD. Again, this is purely hypothesis generating and future studies need to explore this concept further.

The mechanostat theory of Wolf suggests that muscles have a direct impact on bone and muscle contraction and/or stress on the bone, stimulates an increase in bone mass 
$[19,20,35]$. Our findings are in accord with this theory and demonstrate that increasing hip muscle size was positively associated with increased local bone density and muscle strength. A few studies support our findings and suggest that muscle CSA and strength could predict changes in bone health and perhaps bone density $[15,16]$, although some variations do exist. In studies by Edwards and Klein et.al, the association of bone parameters, muscle strength and muscle CSA of the arm and forearm was investigated. Consistent with our results, both studies reported that muscles CSA (forearm and arm) but not muscle strength was a better predictor of bone measures. However, one of the studies measured radial cortical bone area using MRI [16] and the other study used pQCT for assessing bone [15]. In the second study, an association between muscle CSA, bone area, bone mineral content and bone strength but not bone density was found[15]. In comparison to these, we found lower but significant associations between hip muscles and bone density. This may suggest that assessment of actual bone area or bone structure by MRI or pQCT could be more sensitive than bone density measured by DEXA or that relationships alter with increasing age [36].

The relationship between muscle strength and muscle CSA has been investigated [12, $14,15,37]$ and both of these factors are affected by age [16] and disease [12, 38]. Our data is consistent with current literature and expands it showing moderate, consistent associations between hip muscles and muscle strength in older adults (albeit the associations were weaker as compared to other studies). Additionally, not all hip muscles showed an association with strength. Our study includes community-based older adults who were unlikely to be involved in vigorous activity, which may influence this association. Furthermore, we used only one parameter for assessing muscle strength that is mostly applied for measuring hip extensor strength and hence may not equate with other strength measures used in other studies [13, 22]. For instance, Takai et.al reported a strong correlation between quadriceps CSA and knee extension force that was measured by myometer [22].

It is evident that decline in muscle mass has a significant impact on bone mass and strength $[1,3]$. Even though muscle morphology has been explored in biomechanical studies $[39,40]$ it has gain less attention in osteoporosis and/or osteoarthritis (OA). In addition, unlike bone, muscles have some capacity to regenerate [41, 42]. Hence MRI 
studies, like ours, looking into individual muscles can be used for better understanding of changes in muscle morphology in older adults, who are more vulnerable to the development of OA or osteoporosis. Such studies could aid in targeted rehabilitation, which can be applied for management of disease progression and maintenance of bone mass.

\section{Limitations}

This is a cross-sectional study, thus we are unable to determine causal pathways but our results were consistent with the existing data. Bone density was measured using DXA, which provides an areal two-dimensional BMD measure that can be influenced by differences in bone size. However, we adjusted for age and sex, which are reasonable surrogates of bone size differences but did not adjust for other body size related covariates for the reasons outlined above. In addition, the comparison of unilaterally measured BMD and muscle CSA with bilaterally assessed leg strength might have influenced our findings. We measured isometric strength and the associations may differ with alternate strength measures. In this study we were unable to exclude muscle infiltrates and also could not capture the maximal muscle bulk of some muscles due to limited MRI field of view. Furthermore, some muscles were not visible on the MRI scans thus we were unable to assess all hip muscles in each participant or adjust for independent effects of each muscle.

\section{Conclusions}

Overall, hip muscle CSA (especially the hip flexors), and to a lesser extent, muscle strength were positively associated with hip BMD. This data suggests that both higher muscle mass and strength may contribute to the maintenance of bone mass and prevention of disease progress in older adult. 


\section{Acknowledgements}

A special thanks goes to the participants of the TASOAC study. We thank Catrina

Boon and Pip Boon for their contribution in collecting the data for this study.

\section{Declaration of Authors contributions:}

HA, DA, DS, FC, and GJ contributed to the conception and design of the study. HA, LB and GJ contributed in analyses of the data. HA performed the muscle CSA measurements and prepared the first draft of the manuscript. All authors contributed in data interpretation, critical revision and final approval of the manuscript. HA assumes responsibility for the integrity and accuracy of the data.

\section{Funding sources}

This work was supported by the National Health and Medical Research Council of Australia, Tasmanian Community Fund, Masonic Centenary Medical Research Foundation, Royal Hobart Hospital Research Foundation and Arthritis Foundation of Australia.

\section{Competing interests}

The authors state that they have no conflicts of interest.

\section{References:}

1. Blain H, Jaussent A, Thomas E, Micallef JP, Dupuy AM, et al. (2010) Appendicular skeletal muscle mass is the strongest independent factor associated with femoral neck bone mineral density in adult and older men. Exp Gerontol 45:679-684 2. Szulc P, Blaizot S, Boutroy S, Vilayphiou N, Boonen S, et al. (2013) Impaired bone microarchitecture at the distal radius in older men with low muscle mass and grip strength: The STRAMBO study. J Bone Miner Res 28:169-178

3. Lebrasseur NK, Achenbach SJ, Melton LJ, Amin S, Khosla S (2012) Skeletal muscle mass is associated with bone geometry and microstructure and serum insulin- 
like growth factor binding protein-2 levels in adult women and men. $\mathrm{J}$ Bone Miner Res 27:2159-2169

4. Szulc P, Blaizot S, Boutroy S, Vilayphiou N, Boonen S, et al. (2013) Impaired bone microarchitecture at the distal radius in older men with low muscle mass and grip strength: the STRAMBO study. J Bone Miner Res 28:169-178

5. Scott D, Blizzard L, Fell J, Jones G (2012) Prospective study of self-reported pain, radiographic osteoarthritis, sarcopenia progression, and falls risk in communitydwelling older adults. Arthritis Care \& Research 64:30-37

6. Johannesdottir F, Aspelund T, Siggeirsdottir K, Jonsson BY, Mogensen B, et al. (2012) Mid-thigh cortical bone structural parameters, muscle mass and strength, and association with lower limb fractures in older men and women (AGES-Reykjavik Study). Calcif Tissue Int 90:354-364

7. Abellan Van Kan G (2009) Epidemiology and consequences of sarcopenia. JNHA The Journal of Nutrition, Health and Aging 13:708-712

8. Lang T, Cauley JA, Tylavsky F, Bauer D, Cummings S, et al. (2010) Computed tomographic measurements of thigh muscle cross-sectional area and attenuation coefficient predict hip fracture: the health, aging, and body composition study. J Bone Miner Res 25:513-519

9. Kim JH, Choi SH, Lim S, Lim JY, Kim KW, et al. (2013) Thigh muscle attenuation measured by computed tomography was associated with the risk of low bone density in community-dwelling elderly population. Clin Endocrinol 78:512-517

10. Berry P, Teichtahi A, Galevska-Dimitrivska A (2008) Vastus medialis crosssectional area is positively assoicated with patella cartilage and bone volumes in a pain-free community based population. Arthritis Res Ther 10:R143

11. Wluka AE, Ahedi H, Teichtahl AJ, Bennell KL, Tanamas SK, et al. (2012) The cross-sectional area of hip musculature and their relationships to femoral head cartilage in healthy and osteoarthritis individuals. Osteoarthritis Cartilage 20:S184S185

12. Arokoski M, Arokoski J, Haara M, Kankaanpaa M, Vesterinen M, et al. (2002) Hip muscle strength and muscle cross sectional area in men with and without hip osteoarthritis. J Rheumatol 29:2185-2195

13. Masuda K, Kikuhara N, Takahashi H, Yamanaka K (2003) The relationship between muscle cross-sectional area and strength in various isokinetic movements among soccer players. J Sports Sci 21:851-858 
14. Frontera WR, Hughes VA, Fielding RA, Fiatarone MA, Evans WJ, et al. (2000) Aging of skeletal muscle: a 12-yr longitudinal study. J Appl Physiol 88:1321-1326

15. Edwards M, Jameson K, Gregson C, Harvery N, Sayer A, et al. (2012) Muscle size, strength and physical performance as predictors of bone structure in hertfordshire cohort study. Osteoporosis Int 23:S538-S539

16. Klein CS, Allman BL, Marsh GD, Rice CL (2002) Muscle size, strength, and bone geometry in the upper limbs of young and old men. J Gerontol A Biol Sci Med Sci 57:M455-459

17. Revel M, Roux C, Listrat V, Bagheri F, Rabourdin JP, et al. (1993) Cross sectional psoas muscle area is related to trabecular bone mineral density of the lumbar vertebrae in post-menopausal women. Eur J Phys Rehab Med 3:86-90

18. Revel M, Mayoux-Benhamou MA, Rabourdin JP, Bagheri F, Roux C (1993) Oneyear psoas training can prevent lumbar bone loss in postmenopausal women: a randomized controlled trial. Calcif Tissue Int 53:307-311

19. Frost HM (1987) The mechanostat: a proposed pathogenic mechanism of osteoporoses and the bone mass effects of mechanical and nonmechanical agents. Bone Miner 2:73-85

20. Frost HM (1994) Wolff's Law and bone's structural adaptations to mechanical usage: an overview for clinicians. Angle Orthod 64:175-188

21. Stevens JE, Pathare NC, Tillman SM, Scarborough MT, Gibbs CP, et al. (2006) Relative contributions of muscle activation and muscle size to plantarflexor torque during rehabilitation after immobilization. Journal of Orthopaedic Research 24:17291736

22. Takai Y, Ohta M, Akagi R, Kanehisa H, Kawakami Y, et al. (2009) Sit-to-stand test to evaluate knee extensor muscle size and strength in the elderly: a novel approach. J Physiol Anthropol 28:123-128

23. Foley S, Quinn S, Jones G (2010) Pedometer determined ambulatory activity and bone mass: a population-based longitudinal study in older adults. Osteoporos Int 21:1809-1816

24. Scott D, Blizzard L, Fell J, Jones G (2011) Prospective associations between ambulatory activity, body composition and muscle function in older adults. Scand $\mathbf{J}$ Med Sci Sports 21:1600-0838 
25. Rikkonen T, Sirola J, Salovaara K, Tuppurainen M, Jurvelin JS, et al. (2012) Muscle strength and body composition are clinical indicators of osteoporosis. Calcif Tissue Int 91:131-138

26. Di Monaco M, Vallero F, Di Monaco R, Tappero R, Cavanna A (2007) Skeletal muscle mass, fat mass, and hip bone mineral density in elderly women with hip fracture. J Bone Miner Metab 25:237-242

27. Verschueren S, Gielen E, O'Neill TW, Pye SR, Adams JE, et al. (2013) Sarcopenia and its relationship with bone mineral density in middle-aged and elderly European men. Osteoporos Int 24:87-98

28. Stathokostas L, McDonald MW, Little RM, Paterson DH (2013) Flexibility of older adults aged 55-86 years and the influence of physical activity. J Aging Res 743843:19

29. Ming-Sheng Chan, Chen-Fu Huang, Jia-Hao Chang, Thomas Kernozek (2009) Kinematics and Kinetics of Knee and Hip Position of Female Basketball Players during Side-step Cutting with and without Dribbling. Journal of Medical and Biological Engineering, 24:

30. Boyer K. A BGSaATP (2008) Gender differences exist in the hip joint moments of healthy elderly walkers. Journal of biomechanics 41:3360-3365

31. Giphart JE, Stull JD, Laprade RF, Wahoff MS, Philippon MJ (2012) Recruitment and activity of the pectineus and piriformis muscles during hip rehabilitation exercises: an electromyography study. Am J Sports Med 40:1654-1663

32. Ward SR, Winters TM, Blemker SS (2010) The architectural design of the gluteal muscle group: implications for movement and rehabilitation. J Orthop Sports Phys Ther 40:95-102

33. Retchford TH, Crossley KM, Grimaldi A, Kemp JL, Cowan SM (2013) Can local muscles augment stability in the hip? A narrative literature review. J Musculoskelet Neuronal Interact 13:1-12

34. Aung HH, Sakamoto H, Akita K, Sato T (2001) Anatomical study of the obturator internus, gemelli and quadratus femoris muscles with special reference to their innervation. Anat Rec 263:41-52

35. Schoenau E, Neu CM, Beck B, Manz F, Rauch F (2002) Bone mineral content per muscle cross-sectional area as an index of the functional muscle-bone unit. J Bone Miner Res 17:1095-1101 
36. Sukumar D, Schlussel Y, Riedt CS, Gordon C, Stahl T, et al. (2011) Obesity alters cortical and trabecular bone density and geometry in women. Osteoporos Int 22:635645

37. Stephen M. Roth FMI, Greg F Martel (2002) Muscle size response to strength training in young and older men and women Journal of American Geriatrics Society 49:5

38. Anton Rasch AHB, Nils Dalen and Hans E Berg (2007) Reduced muscle radiological density, cross-sectional area and strength of major hip and knee muscles in 22 patients with hip osteoarthritis. Acta Orthopaedica 78:505-510

39. Berg HE, Eiken O, Miklavcic L, Mekjavic IB (2007) Hip, thigh and calf muscle atrophy and bone loss after 5-week bedrest inactivity. Eur J Appl Physiol 99:283-289 40. Miokovic T, Armbrecht G, Felsenberg D, Belavy DL (2012) Heterogeneous atrophy occurs within individual lower limb muscles during 60 days of bed rest. J Appl Physiol 113:1545-1559

41. Hubal MJG-D, Heather; Thomson, Paul D (2005) Variability in Muscle Size and Strength Gain after Unilateral Resistance Training. Medicine \& Science in Sports \& Exercise: $37: 8$

42. Anton Rasch AHB, Nils Dalen and Hans E Berg (2007) Reduced muscle radiological density, cross-sectional area, and strenght of major hip and knee muscles in 22 patients with hip osteoarthritis. Acta Orthopaedica 78:505-510

\section{Figure legends:}

Figure 1: Measurement of hip muscle CSA using OsiriX imaging software. (Iliopsoas and quadratus femoris CSA shown as examples)

Figure 2: Demonstration of the leg strength measurement in TASOAC. 
Table 1: Hip muscles anatomical landmark chart.

Hip Muscle Anatomical Landmark

\section{Hip extensor}

Gluteus Maximus First appearance of the femoral head cartilage in sagittal section

\section{Hip rotators}

Obturator externus First appearance of the femoral head cartilage in sagittal section

Gemelli First appearance of the femoral neck. 
Quadratus femoris Round head of the femur is no longer visible in the sagittal section

Piriformis First appearance of the bony femoral head in sagittal section

\section{Hip flexors}

\begin{tabular}{ll}
\hline Pectineus & $\begin{array}{l}\text { First appearance of the femoral head cartilage in } \\
\text { sagittal section (adjacent to obturator externus) }\end{array}$ \\
Tliopsoas & Total visibility of the femoral fovea in axial \\
& section \\
Sartorius & First appearance of the femoral neck in sagittal \\
& section
\end{tabular}

Table 2: Characteristics of the subjects

\begin{tabular}{lcc}
\hline Characteristics & $\begin{array}{c}\text { Men } \\
(\mathrm{n}=167)\end{array}$ & $\begin{array}{c}\text { Women } \\
(\mathrm{n}=158)\end{array}$ \\
\hline Age $(\mathrm{yrs})$. & $64.04(7.47)$ & $63.26(6.60)$ \\
BMI $\left(\mathrm{kg} / \mathrm{m}^{2}\right)$ & $27.50(3.91)$ & $28.13(5.23)$ \\
Total hip BMD $\left(\mathrm{g} / \mathrm{cm}^{2}\right)$ & $1.02(0.13)$ & $0.90(0.13)$ \\
Femoral neck BMD $\left(\mathrm{g} / \mathrm{cm}^{2}\right)$ & $0.80(0.11)$ & $0.74(0.11)$
\end{tabular}


Spine BMD $\left(\mathrm{g} / \mathrm{cm}^{2}\right)$

Leg strength $(\mathrm{kg})$

Steps per day

Gluteus Maximus CSA $\left(\mathrm{cm}^{2}\right)$

Obturator Externus CSA $\left(\mathrm{cm}^{2}\right)$

Gemelli CSA $\left(\mathrm{cm}^{2}\right)$

Quadratus femoris CSA $\left(\mathrm{cm}^{2}\right)$

Pectineus CSA $\left(\mathrm{cm}^{2}\right)$

Piriformis CSA $\left(\mathrm{cm}^{2}\right)$

Sartorius CSA $\left(\mathrm{cm}^{2}\right)$

Iliopsoas CSA $\left(\mathrm{cm}^{2}\right)$

Data presented as means (sd).

$\mathrm{CSA}=$ cross-sectional area.

$\mathrm{BMD}=$ bone mineral density .

$\begin{array}{cc}1.07(0.17) & 0.96(0.15) \\ 135.32(45.7) & 63.11(28.9) \\ 8268(3703) & 7384(3234) \\ 51.40(13.6) & 42.20(8.05) \\ 9.31(1.83) & 7.11(1.13) \\ 4.33(1.00) & 3.50(0.93) \\ 7.20(2.46) & 5.92(1.70) \\ 10.31(2.09) & 7.71(1.84) \\ 4.92(1.52) & 3.87(1.24) \\ 10.24(3.67) & 7.71(2.24) \\ 10.08(1.81) & 7.63(1.48)\end{array}$

$0.96(0.15)$

$42.20(8.05)$

$.11(1.13)$

7.71 (1.84)

3.87 (1.24)

$7.63(1.48)$ 
Table 3: Cross-sectional relationship between hip muscle CSA and bone density at the hip, neck and spine

\begin{tabular}{|c|c|c|c|c|}
\hline Study factor & $\mathrm{N}$ & $\begin{array}{c}\text { Total hip BMD }\left(\mathrm{g} / \mathrm{cm}^{2}\right) \\
\text { Standardized } \beta \text { eta }(95 \% \mathrm{CI}) \\
\text { (Adjusted })^{\dagger}\end{array}$ & $\begin{array}{c}\text { Femoral neck BMD }\left(\mathrm{g} / \mathrm{cm}^{2}\right) \\
\text { Standardized Beta }(95 \% \mathrm{CI}) \\
\text { (Adjusted) }^{\dagger}\end{array}$ & $\begin{array}{c}\text { Spine BMD }\left(\mathrm{g} / \mathrm{cm}^{2}\right) \\
\text { Standardized } \beta \text { eta }(95 \% \mathrm{CI}) \\
\text { (Adjusted) }{ }^{\dagger}\end{array}$ \\
\hline \multicolumn{5}{|l|}{ Hip extensor } \\
\hline $\begin{array}{l}\text { Gluteus maximus }\left(\mathrm{cm}^{2}\right) \\
\text { Hip rotators }\end{array}$ & 84 & $0.01(-0.28,0.31)$ & $-0.00(-0.35,0.27)$ & $-0.03(-0.19,0.13)$ \\
\hline Obturator Externus $\left(\mathrm{cm}^{2}\right)$ & 219 & $0.18(0.00,0.35)$ & $0.26(0.10,0.40)$ & $0.05(-0.07,0.16)$ \\
\hline Gemelli $\left(\mathrm{cm}^{2}\right)$ & 130 & $0.04(-0.12,0.21)$ & $0.03(-0.14,0.20)$ & $0.20(0.08,0.33)$ \\
\hline Quadratus femoris $\left(\mathrm{cm}^{2}\right)$ & 236 & $0.19(0.07,0.31)$ & $0.16(0.03,0.30)$ & $0.04(-0.06,0.14)$ \\
\hline Piriformis $\left(\mathrm{cm}^{2}\right)$ & 240 & $0.11(-0.02,0.23)$ & $0.06(-0.06,0.20)$ & $0.07(-0.02,0.17)$ \\
\hline \multicolumn{5}{|l|}{ Hip flexors } \\
\hline Pectineus $\left(\mathrm{cm}^{2}\right)$ & 153 & $0.22(0.06,0.37)$ & $0.21(0.05,0.36)$ & $0.09(-0.06,0.23)$ \\
\hline Men & 87 & $0.18(-0.05,0.42)$ & $0.14(-0.10,0.37)$ & - \\
\hline Women & 66 & $0.27(0.05,0.56)$ & $0.31(0.09,0.52)$ & - \\
\hline Interaction ( $p$ value) & & $p=0.04$ & $p=0.01$ & \\
\hline Sartorius $\left(\mathrm{cm}^{2}\right)$ & 145 & $0.22(0.06,0.37)$ & $0.26(0.09,0.42)$ & $0.05(-0.06,0.17)$ \\
\hline Men & 55 & $0.13(-0.15,0.41)$ & $0.09(-0.19,0.37)$ & - \\
\hline Women & 90 & $0.29(0.09,0.48)$ & $0.41(0.23,0.59)$ & - \\
\hline Interaction ( $p$ value) & & $p=0.06$ & $p=0.001$ & \\
\hline Iliopsoas $\left(\mathrm{cm}^{2}\right)$ & 215 & $0.20(0.05,0.36)$ & $0.20(0.03,0.35)$ & $0.03(-0.08,0.15)$ \\
\hline
\end{tabular}

Bold face indicates statistically significant. Independent variable: hip muscles. Dependent variable: BMD at hip, neck and spine.

$\uparrow$ Adjusted for age, sex and steps per day. Additionally, models for spine BMD have been adjusted for hip BMD

$\beta e t a$ coefficient (standardized) represents cross-sectional increase in bone density at hip, neck and spine BMD with per unit increase in CSA of hip muscles. 
Table 4: Cross-sectional associations between hip muscle CSA and muscle strength.

\begin{tabular}{|c|c|c|}
\hline Study factor & $\mathrm{N}$ & $\begin{array}{c}\text { Standardized } \beta \text { eta } \\
\text { (95\% CI) } \\
\text { (Adjusted) }{ }^{\dagger}\end{array}$ \\
\hline \multicolumn{3}{|l|}{ Hip extensor } \\
\hline Gluteus maximus $\left(\mathrm{cm}^{2}\right)$ & 81 & $0.11(-0.01,0.23)$ \\
\hline \multicolumn{3}{|l|}{ Hip rotators } \\
\hline Obturator externus $\left(\mathrm{cm}^{2}\right)$ & 206 & $0.16(0.05,0.28)$ \\
\hline Gemelli $\left(\mathrm{cm}^{2}\right)$ & 125 & $0.08(-0.06,0.21)$ \\
\hline Quadratus femoris $\left(\mathrm{cm}^{2}\right)$ & 222 & $0.14(0.04,0.24)$ \\
\hline Piriformis $\left(\mathrm{cm}^{2}\right)$ & 226 & $0.12(0.02,0.23)$ \\
\hline \multicolumn{3}{|l|}{ Hip flexors } \\
\hline Pectineus $\left(\mathrm{cm}^{2}\right)$ & 144 & $0.16(0.04,0.28)$ \\
\hline Sartorius $\left(\mathrm{cm}^{2}\right)$ & 135 & $0.12(0.01,0.23)$ \\
\hline Iliopsoas $\left(\mathrm{cm}^{2}\right)$ & 205 & $0.24(0.12,0.37)$ \\
\hline Men & 95 & $0.32(0.15,0.49)$ \\
\hline Women & 110 & $0.17(0.01,0.35)$ \\
\hline Interaction ( $p$ value) & & $p=0.02$ \\
\hline \multicolumn{3}{|c|}{$\begin{array}{l}\text { Bold face indicates statistically significant } \\
\text { Independent variable: hip muscles. Dependent variable: leg strength. } \\
\mathrm{n}=\text { number o observations. } \\
\dagger \text { Adjusted for age, sex and steps per day. } \\
\text { Beta coefficient (standardized) represents cross-sectional increase in leg strength with per uni } \\
\text { increase in CSA of hip muscles. }\end{array}$} \\
\hline
\end{tabular}




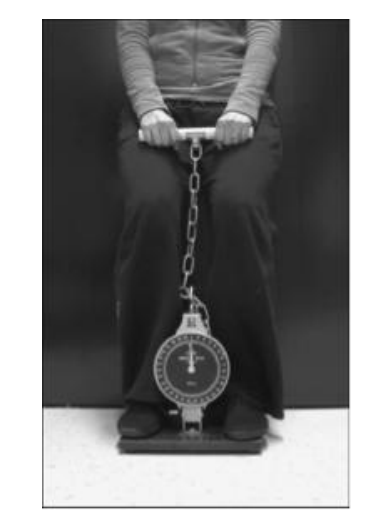

